\title{
Real-time homogenous translucent material editing
}

\author{
Kun $\mathrm{Xu}^{1} \quad$ Yue Gao $^{1} \quad$ Yong $\mathrm{Li}^{1} \quad \mathrm{Tao} \mathrm{Ju}^{2} \quad$ Shi-Min $\mathrm{Hu}^{1}$ \\ ${ }^{1}$ Tsinghua University \\ 2 Washington University in St. Louis
}

\begin{abstract}
This paper presents a novel method for real-time homogenous translucent material editing under fixed illumination. We consider the complete analytic BSSRDF model proposed by Jensen et al. [JMLH01], including both multiple scattering and single scattering. Our method allows the user to adjust the analytic parameters of BSSRDF and provides high-quality, real-time rendering feedback. Inspired by recently developed Precomputed Radiance Transfer (PRT) techniques, we approximate both the multiple scattering diffuse reflectance function and the single scattering exponential attenuation function in the analytic model using basis functions, so that re-computing the outgoing radiance at each vertex as parameters change reduces to simple dot products. In addition, using a non-uniform piecewise polynomial basis, we are able to achieve smaller approximation error than using bases adopted in previous PRT-based works, such as spherical harmonics and wavelets. Using hardware acceleration, we demonstrate that our system generates images comparable to [JMLHO1] at real-time frame-rates.
\end{abstract}

Categories and Subject Descriptors (according to ACM CCS): I.3.3 [Computer Graphics]: Display algorithms; I.3.7 [Computer Graphics]: Color, shading, shadowing, and texture

\section{Introduction}

Translucent materials can be found almost everywhere in our daily life, such as marble, milk, bread, wax, paper, jade, etc. Traditionally, computer rendering of $3 \mathrm{D}$ objects is based on the BRDF (Bidirectional reflectance distribution function $\left[\mathrm{NRH}^{*} 77\right]$ ) model, which assumes that light enters and exits surface at the same point. However, BRDF cannot model translucent materials well as they exhibit significant light scattering below surface, which is known as subsurface scattering. Subsurface scattering effects can be simulated accurately using a number of offline techniques, but it may take hours to render a frame. Jensen et al. [JMLH01] proposed an analytic BSSRDF (Bidirectional subsurface reflectance distribution function $\left[\mathrm{NRH}^{*} 77\right]$ ) model approximation for subsurface scattering, which speeds up the rendering by 2 orders of magnitude (i.e., from hours to minutes). This breakthrough work has led to the popularity of translucent materials in 3D applications, and a great demand has arisen for interactive translucent material design.

In this paper, we present a real-time rendering system for homogenous translucent material editing. Our system considers the complete analytic BSSRDF model proposed by
Jensen et al. [JMLH01] that includes both multiple scattering and single scattering effects. We allow the user to dynamically change the parameters of the analytic model (e.g., the scattering coefficient, the absorption coefficient, and the phase function) under fixed environment lighting while providing high-quality rendering feedback at real-time framerates. Using the system, a designer can easily adjust the look of a translucent material, ranging from glassy, jade to porcelain, as well as lighting effects such as forward and backward scattering.

Our work is inspired by Precomputed Radiance Transfer (PRT), first introduced by Sloan et al. [SKS02]. PRTbased methods reduce the costly integrals in lighting computation to simple dot products by approximating environment light and light transport functions using weighted sum of basis functions. In our work, we approximate the multiple scattering diffuse reflectance function and the single scattering exponential attenuation function in the analytic BSSRDF model using a non-uniform piecewise polynomial basis. Prior to each editing session, the transport functions due to these basis functions can be precomputed and efficiently stored. During each session as the user changes the para- 
meters to the analytic model, the outgoing radiance at each vertex is obtained as a simple dot product between the transport functions and the basis function coefficients, which can be quickly obtained once at each rendering frame.

Contributions In the context of recent works on interactive rendering of homogenous translucent materials [LGB* 03 , $\left.\mathrm{MKB}^{*} 03, \mathrm{HV} 04\right]$ and on using PRT for rendering acceleration [SKS02, NRH03, WTL05, BAOR06], we see this work make the following contributions:

- We extend the PRT techniques onto Jensen's analytical BSSRDF model, and show that the outgoing radiance under fixed environment lighting and varying parameters (e.g., the scattering coefficient, the absorption coefficient, and the phase function) can be efficiently computed using basis function approximation of the multiple scattering diffuse reflectance function and the single scattering exponential attenuation function. .

- We show that a well-designed non-uniform piecewise polynomial basis better approximates both the multiple and single scattering components in the analytical BSSRDF model than previously used bases in PRT methods, such as spherical harmonic bases and wavelet bases.

- Based on the theoretical results, we present an interactive system for editing homogenous translucent materials. Through precomputation and hardware acceleration, the system provides real-time, high-quality rendering of both multiple and single scattering effects with dynamically changing BSSRDF parameters.

\section{Related Work}

Translucent Materials: Subsurface scattering effects can be simulated using a number of offline techniques [HK93, Sta95, JC98, DEL*99, PH00, LPT05]. While capable of producing impressive renderings, these methods are slow and typically require several hours to render one frame. To improve speed, Jensen et al. [JMLH01] proposed an efficient solution for homogenous materials. They formulated the complete BSSRDF as the sum of a single scattering and a multiple scattering component, and approximated the multiple scattering using a dipole diffusion approximation (see detailed discussion in Section 3). Although Jensen's improvement performs 2 orders of magnitude faster than previous Monte Carlo path tracing techniques, it still takes minutes to render a frame. Later, Jensen et al. [JB02] proposed a two pass hierarchical method to accelerate the computation of diffusion approximation, which further reduces the rendering time to seconds per frame.

To achieve interactive or even real-time rendering of homogeneous materials, the work of Lensch et al. [LGB*03] and Hao et al. [HV04] approximated Jensen's BSSRDF model based on the observation that multiple scattering falls off exponentially. However, these work do not permit interactive editing of material parameters. Mertens et al. $\left[\mathrm{MKB}^{*} 03\right]$ proposed a hierarchical boundary element method for interactively rendering deformable translucent meshes. Their method allows the user to change light, view, geometry as well as material parameters at interactive framerates. However, their method is limited to handling only multiple scattering.

Precomputed Radiance Transfer: Precomputed radiance transfer framework for static scenes was first introduced by Sloan et al. [SKS02], and has been extended in a number of works to accelerate rendering of low-frequency [SKS02, SLS05] or all-frequency [NRH03, TS06] effects. In particular, Ben-Artzi et al. [BAOR06] proposed a real-time BRDF editing method based on PRT, where the BRDF functions are approximated by a linear combination of Daubechies wavelet basis. To further address subsurface scattering effects, PRT has been extended to include Jensen's analytic BSSRDF model by Sloan et al. [SHHS03] and by Wang et al. [WTL05]. While the former uses a non-physically based approximation (a glossy BRDF) to the single scattering components in the BSSRDF model, the latter considers the complete model with both multiple and single scattering components and decomposes the phase function into view- and light- independent parts in order to achieve viewdependent single scattering effects. In both methods, basis functions (spherical harmonics basis [SKS02] or wavelet basis [WTL05]) are used to approximate the environment lighting, so that interactive or real-time rendering is achieved under dynamically changing lighting. Unfortunately, these works do not allow efficient change of the analytic BSSRDF parameters.

\section{Background}

In this section, we review the necessary background of the analytic BSSRDF model proposed by Jensen et al. [JMLH01]. The analytic BSSRDF describes the outgoing radiance at point $x_{o}$ in direction $\omega_{o}$ as the sum of a multiple scattering component $\left(L_{d}\right)$ and a single scattering component $\left(L_{O}^{(1)}\right)$ :

$$
L_{o}\left(x_{o}, \omega_{o}\right)=L_{d}\left(x_{o}, \omega_{o}\right)+L_{o}^{(1)}\left(x_{o}, \omega_{o}\right)
$$

The multiple scattering component is approximated using dipole source approximation as:

$$
L_{d}\left(x_{o}, \omega_{o}\right)=\frac{1}{\pi} F_{t}\left(\eta, \omega_{o}\right) \int_{A} R_{d}\left(\left\|x_{o}-x_{i}\right\|\right) I\left(x_{i}\right) d A\left(x_{i}\right)
$$

where the incoming irradiance of point $x_{i}, I\left(x_{i}\right)$, has the form

$$
I\left(x_{i}\right)=\int_{2 \pi} F_{t}\left(\eta, \omega_{i}\right) L\left(x_{i}, \omega_{i}\right)\left(n_{i} \cdot \omega_{i}\right) d \omega_{i},
$$




\begin{tabular}{ll}
\hline$R_{d}$ & Diffuse reflectance function \\
$E$ & Exponential attenuation function \\
$F_{t}$ & Fresnel transmittance \\
$\eta$ & Relative index of refraction \\
$\sigma_{s}$ & Scattering coefficient \\
$\sigma_{a}$ & Absorption coefficient \\
$g$ & Mean cosine of the scattering angle \\
$p$ & Normalized phase function \\
$\sigma_{t}=\sigma_{a}+\sigma_{s}$ & extinction coefficient \\
$\sigma_{s}^{\prime}=(1-g) \sigma_{s}$ & reduced scattering coefficient \\
$\sigma_{t}^{\prime}=\sigma_{a}+\sigma_{s}^{\prime}$ & reduced extinction coefficient \\
$\alpha^{\prime}=\sigma_{s}^{\prime} / \sigma_{t}^{\prime}$ & reduced albedo \\
$\sigma_{t r}=\sqrt{3 \sigma_{a} \sigma_{t}^{\prime}}$ & effective extinction coefficient \\
& \\
$F_{d r}=-1.440 / \eta^{2}$ & $+0.710 / \eta+0.668+0.0636 \eta$ \\
$A=\left(1+F_{d r}\right) /\left(1-F_{d r}\right)$ & \\
$z_{r}=1 / \sigma_{t}^{\prime}, z_{v}=z_{r}(1+4 A / 3)$ \\
$r=\left\|x_{i}-x_{o}\right\|$ & \\
$d_{r}=\sqrt{r^{2}+z_{r}^{2}}, d_{v}=\sqrt{r^{2}+z_{v}^{2}}$
\end{tabular}

Table 1: Selected Symbols

and the diffuse reflectance function $R_{d}$ is computed as

$$
R_{d}(r)=\frac{\alpha^{\prime}}{4 \pi}\left[z_{r}\left(\sigma_{t r}+\frac{1}{d_{r}}\right) \frac{e^{-\sigma_{t r} d_{r}}}{d_{r}^{2}}+z_{v}\left(\sigma_{t r}+\frac{1}{d_{v}}\right) \frac{e^{-\sigma_{t r} d_{v}}}{d_{v}^{2}}\right]
$$

Here, $L\left(x_{i}, \omega_{i}\right)$ is the incident radiance of point $x_{i}$ in the direction $\omega_{i}, n_{i}$ is the normal direction at $x_{i}$, and the other symbols are explained in Table 1.

The single scattering component is defined as

$$
L_{o}^{(1)}\left(x_{o}, \omega_{o}\right)=\sigma_{s} \int_{2 \pi} \int_{0}^{\infty} F p(\theta) E\left(s_{i}^{\prime}+s\right) L\left(x_{i}, \omega_{i}\right) d s d \omega_{i}
$$

where $\theta=\cos ^{-1}\left(\omega_{i}^{\prime} \cdot \omega_{o}^{\prime}\right)$ is the angle between $\omega_{i}^{\prime}$ and $\omega_{o}^{\prime}$, $F=F_{t}\left(\eta, \omega_{o}\right) F_{t}\left(\eta, \omega_{i}\right)$ is the product of the two fresnel transmittances, $\omega_{i}^{\prime}$ and $\omega_{o}^{\prime}$ are the refracted incoming and outgoing directions, $s_{i}^{\prime}$ and $s$ are the scattering path along $\omega_{i}^{\prime}$ and $\omega_{o}^{\prime}$, and the exponential attenuation function $E(r)$ is computed by:

$$
E(r)=e^{-\sigma_{t} r}
$$

Assuming that the shadow ray does not refract at $x_{i}$, we use an approximation of the refracted distance $s_{i}^{\prime}$ by

$$
s_{i}^{\prime}=s_{i} \frac{\left|\omega_{i} \cdot n_{i}\right|}{\sqrt{1-\left(\frac{1}{\eta}\right)^{2}\left(1-\left|\omega_{i} \cdot n_{i}\right|^{2}\right)}}
$$

where $s_{i}$ is the observed distance as if the incident ray is not refracted.

In summary, the parameters for the analytic BSSRDF model include the absorption coefficient $\sigma_{a}$, the scattering coefficient $\sigma_{s}$, the phase function $p$, and the relative index of refraction $\eta$. In the following sections $(3,4,5)$, we assume that both $\eta$ and illumination $L\left(x_{i}, \omega_{i}\right)$ are fixed (so that the incoming irradiance $I\left(x_{i}\right)$ is fixed), and we show that the outgoing radiance $L_{o}$ in Equation 1 can be efficiently approximated when the other three parameters vary.

\section{Approximating Analytic BSSRDF}

Following the PRT rational, we approximate the analytic BSSRDF model using basis functions. We will show that, once the multiple scattering diffuse reflectance function $R_{d}$ and the single scattering exponential attenuation function $E$ are represented as linear combinations of basis functions, the outgoing radiance $L_{o}$ in Equation 1 reduces to dot products between the coefficients of the basis functions and precomputable components known as transport functions.

Multiple Scattering: Let the diffuse reflectance function $R_{d}$ be approximated by specific basis functions $B_{j}^{R}$ with coefficients $c_{j}^{R}$ (the choice of such functions and the computation of their coefficients will be discussed in Section 5):

$$
R_{d}(r) \approx \sum_{j} c_{j}^{R} B_{j}^{R}(r)
$$

Substituting Equation 8 into Equation 2 yields:

$$
L_{d}\left(x_{o}, \omega_{o}\right) \approx \frac{1}{\pi} F_{t}\left(\eta, \omega_{o}\right) \sum_{j} c_{j}^{R} T_{j}\left(x_{o}\right)
$$

where

$$
T_{j}\left(x_{o}\right)=\int_{A} B_{j}^{R}\left(\left\|x_{o}-x_{i}\right\|\right) I\left(x_{i}\right) d A\left(x_{i}\right)
$$

Note that Equation 9 only involves the dot product of the coefficients $c_{j}^{R}$ of the basis functions and the $T_{j}$. For convenience, we refer to $T_{j}$ as a transport function. The key observation is that $T_{j}$ is independent of the three analytic BSSRDF parameters $\left(\sigma_{a}, \sigma_{s}\right.$, and $\left.p\right)$, and hence can be precomputed prior to editing (see Section 6).

Single Scattering: As denoted in Wang et al. [WTL05], precomputing single scattering according to Equation 5 is difficult since both the integration path and the phase function depend on the view direction, and enumerating each possible view direction during precomputation is infeasible. To fix the integration path, we adopt the approach in [WTL05] by using the negative normal direction of each vertex $x_{0}$. To precompute the phase function, Wang et al. [WTL05] decomposed the 4D phase function to several separated 2D lightand view- maps using singular value decomposition (SVD). However, a texture is required to store each map. Here we introduce a novel, more compact decomposition of the phase function where both the light- and view- maps are analytical functions and no longer need to be stored as textures.

We observe that the $\cos \theta$ in the typical Eddington phase function $p(\theta)=(1+3 g \cos \theta) /(4 \pi)$ ( $g$ is the mean cosine of scattering angle) is the dot product of the refracted light direction $\omega_{i}^{\prime}$ and the refracted view direction $\omega_{o}^{\prime}$. Hence $p$ 
can be re-written as a dot product:

$$
p\left(\omega_{i}^{\prime}, \omega_{o}^{\prime}\right)=\vec{G}\left(\omega_{i}^{\prime}\right) \cdot \vec{H}\left(\omega_{o}^{\prime}\right)
$$

Let $\omega_{i}^{\prime}=\left(a_{0}, a_{1}, a_{2}\right)$, and $\omega_{o}^{\prime}=\left(b_{0}, b_{1}, b_{2}\right)$, then $\vec{G}\left(\omega_{i}^{\prime}\right)=$ $\left(1, a_{0}, a_{1}, a_{2}\right)$ and $\vec{H}\left(\omega_{o}^{\prime}\right)=\left(1,3 g b_{0}, 3 g b_{1}, 3 g b_{2}\right) /(4 \pi)$ are 4 -tuples involving only $\omega_{i}^{\prime}$ or $\omega_{o}^{\prime}$. Substituting Equation 11 into Equation 5, the outgoing radiance of point $x_{o}$ due to single scattering $L_{O}^{(1)}$ becomes

$$
\begin{aligned}
& L_{o}^{(1)}\left(x_{o}, \omega_{o}\right)=F t\left(\eta, \omega_{o}\right) \sigma_{s} \vec{H}\left(\omega_{o}^{\prime}\right) . \\
& \quad \int_{2 \pi} \int_{0}^{\infty} \vec{G}\left(\omega_{i}^{\prime}\right) F t\left(\eta, \omega_{i}\right) E\left(s_{i}^{\prime}+s\right) L\left(x_{i}, \omega_{i}\right) d s d \omega_{i}(12
\end{aligned}
$$

where the integral involves only the view-independent portion of the phase function.

To approximate $L_{o}^{(1)}$, we let the single scattering exponential attenuation function $E$ in Equation 6 be approximated by some basis functions $B_{j}^{E}$ with coefficients $c_{j}^{E}$ (to be discussed in Section 5):

$$
E(r) \approx \sum_{j} c_{j}^{E} B_{j}^{E}(r)
$$

Substituting Equation 13 into Equation 12 yields a dotproduct approximation of single scattering:

$$
L_{o}^{(1)}\left(x_{o}, \omega_{o}\right) \approx F t\left(\eta, \omega_{o}\right) \sigma_{S} \vec{H}\left(\omega_{o}^{\prime}\right) \cdot \sum_{j} c_{j}^{E} \overrightarrow{S_{j}}\left(x_{o}\right)
$$

where

$\overrightarrow{S_{j}}\left(x_{o}\right)=\int_{2 \pi} \int_{0}^{\infty} \vec{G}\left(\omega_{i}^{\prime}\right) B_{j}^{E}\left(s_{i}^{\prime}+s\right) L\left(x_{i}, \omega_{i}\right) F t\left(\eta, \omega_{i}\right) d s d \omega_{i}$

As in multiple scattering, we refer to $\overrightarrow{S_{j}}$ as a transport function. Thanks to the decomposition of the phase function, $\overrightarrow{S_{j}}$ is independent of viewing direction as well as the three analytic BSSRDF parameters ( $\sigma_{a}, \sigma_{s}$ and the phase function), and can be easily precomputed and stored (see Section 6).

\section{Piecewise Polynomial Basis}

A variety of basis functions, including spherical harmonics and wavelets, have been used and shown effective in PRT-based methods. Nonetheless, our task is to find an appropriate basis that can approximate the diffuse reflectance function $R_{d}$ and the exponential attenuation function $E$ accurately and smoothly using only a small number of basis functions. In particular, since both $R_{d}$ and $E$ are strong attenuation functions, they cannot be accurately approximated by a small number of low frequency basis such as spherical harmonics (in 1D, spherical harmonics is Fourier basis function). For wavelet bases, [BAOR06] points out that the standard non-linear wavelet bases are not suitable for material editing, as they ignore small coefficients by definition and would provide no feedback for small changes in the parameters. On the other hand, a full wavelet basis would require a large number of basis functions to capture the attenuated portions of $R_{d}$ and $E$ (see comparison in Section 7).
Here we propose a piecewise polynomial basis defined on non-uniform intervals that are better adapted to approximate strong attenuation functions.

Piecewise polynomial basis has been widely investigated and used in numerical analysis and related applications. In computer graphics, piecewise polynomial approximation has been used in importance sampling techniques [LRR05]. Specifically, we consider a domain $[a, b]$ and a sequence of $m+1$ points $r_{0}, r_{1}, \ldots, r_{m}$ (called knots) satisfying $a=r_{0}<$ $r_{1}<\ldots<r_{m}=b$, which partition the domain into $m$ intervals $I_{k}=\left[r_{k}, r_{k+1}\right]$ for $k \in[0, m-1]$. A degree- $(n-1)$ piecewise polynomial approximation of a function $F$ defined on $[a, b]$ has the form

$$
F(r) \approx P(r)=\sum_{j=0}^{j<n \cdot m} c_{j} B_{j}(r)
$$

where $c_{j}$ are $m \times n$ polynomial coefficients, and $B_{j}$ are the piecewise polynomial basis defined as:

$$
B_{i+k n}(r)=\left\{\begin{array}{l}
r^{i}, r \in I_{k} \\
0, r \notin I_{k}
\end{array}\right.
$$

for $i \in[0, n-1]$ and $k \in[0, m-1]$. In addition, we require that $P$ is a polynomial of degree $\leq(n-1)$ on each interval $I_{k}$ and is $C^{(n-1)}$-continuous at each knot $r_{k}$.

Projection: To determine the polynomial coefficients $c_{j}$ in Equation 16 given a function $F$ (in our case, $R_{d}$ or $E$ ), its domain $[a, b]$, and intervals $I_{k}$, we formulate a constrained discrete minimization problem

$$
\text { minimize } \sum_{\tau \in[a, b]}(F(\tau)-P(\tau))^{2}
$$

where $\tau$ are samples on the domain $[a, b]$ and $P$ is constrained to be $C^{(n-1)}$-continuous at each knot $r_{k+1}$, that is,

$$
P_{k}^{i}\left(r_{k+1}\right)=P_{k+1}^{i}\left(r_{k+1}\right)
$$

for $i \in[0, n-1]$ and $k \in[0, m-2]$. Here $P_{k}^{i}(r)$ is the $i$-th order derivative of the degree- $(n-1)$ polynomial of $P$ restricted to interval $I_{k}$, i.e., $\sum_{i=0}^{n-1} c_{i+k n} r^{i}$. Since the minimization target in Equation 18 is quadratic and the constraint is linear, the problem could be efficiently solved by a least square solver. In our implementation, we uniformly choose 10 samples $(\tau$ in Equation 18) in each interval. With a small dimension of the linear system (usually involving tens of variables), computing the polynomial coefficients $c_{j}$ costs less than 1 millisecond.

Interval Partition: Ideally, to achieve best approximation of $F$ using piecewise polynomials, we would like to find the knots $r_{k}$ and the coefficients $c_{j}$ that minimize Equation 18. However, such minimization is non-trivial due to the involvement of two types of variables. To simplify the problem, we find knots by choosing a fixed set of coefficients. In our method, we consider such coefficients that result in a 


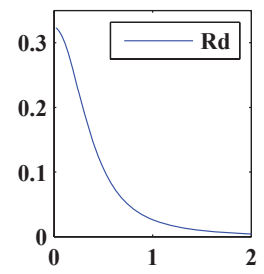

(a) Rd

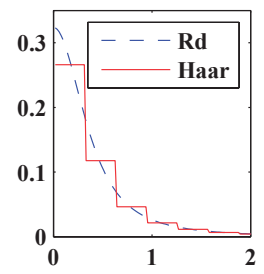

(b) Haar 64

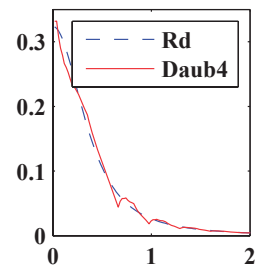

(c) Daub4 64

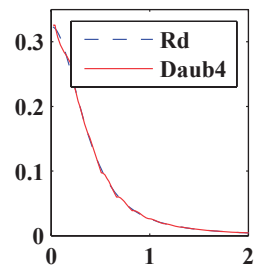

(d) Daub4 128

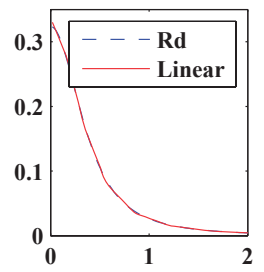

(e) Linear 32

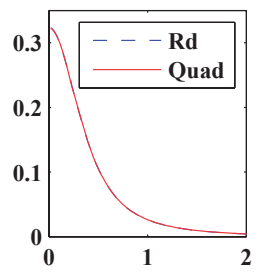

(f) Quadric 48

Figure 1: Comparison of the diffuse reflectance function $R_{d}(r)$ with parameters $\sigma_{s}=2.0, \sigma_{a}=0.1$ (a) and approximations using Haar wavelet with 64 coefficients (b), Daubechies 4 wavelet with 64 coefficients (c) and 128 coefficients (d), piecewise linear basis with 32 coefficients (e), and piecewise quadratic basis with 48 coefficients (f). Notice that piecewise linear basis with 32 coefficients achieves similar approximation accuracy as Daubechies 4 wavelets with 128 coefficients.
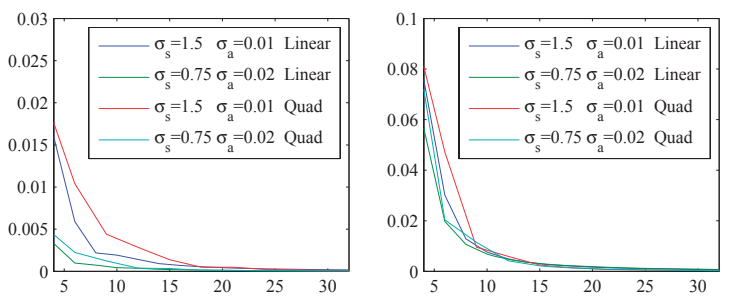

Figure 2: RMS error of approximating $R_{d}$ (left) and $E$ (right) with linear or quadratic basis under different scattering and absorption coefficients $\left(\sigma_{a}, \sigma_{s}\right)$, plotted with the increase of the number of coefficients.

piecewise linear polynomial interpolating $F$ at each knot:

$$
P(r)=\frac{\left(r_{k+1}-r\right) F\left(r_{k}\right)+\left(r-r_{k}\right) F\left(r_{k+1}\right)}{r_{k+1}-r_{k}}
$$

where $r_{k} \leq r<r_{k+1}$ and $k \in[0, m-1]$. Given a userspecified number of intervals $m$ and the $P$ defined above, the knots $r_{k}$ can be found by solving the minimization problem in Equation 18 using discrete dynamic programming. Furthermore, to avoid performing this computation during realtime rendering, we precompute the knots before the editing session using a representative $F$. In our implementation, the representative $F$ is chosen as the average of three $R_{d}(r)$ with parameters $\sigma_{s}=0.2, \sigma_{a}=0.001, \sigma_{s}=2, \sigma_{a}=$ 0.02 , and $\sigma_{s}=10, \sigma_{a}=0.2$ for multiple scattering, and as $\frac{1}{3}\left(e^{-0.2}+e^{-2}+e^{-10}\right)$ for single scattering.

\section{Interactive Editing System}

Using piecewise polynomial basis approximation of the analytic BSSRDF model, we have developed an interactive system for editing homogeneous translucent materials. Here we detail the implementation in the two stages of an editing session: precomputation and real-time rendering.

\subsection{Precomputation}

Given a static scene under fixed environment lighting, we precompute prior to each editing session the two transport functions at each vertex, which are the $T_{j}$ in Equation 10 for multiple scattering and the $\overrightarrow{S_{j}}$ in Equation 15 for single scattering.

For multiple scattering, we first evenly and densely distribute a set of sample points and use ray tracing to compute the incoming irradiance at each sample point. Next, we compute $T_{j}\left(x_{o}\right)$ for each vertex $x_{o}$ by an integration over all the sample points using Equation 10. To make the computation more efficient, we observe that the piecewise polynomial ba$\operatorname{sis} B_{j}^{R}$ is non-zero only within a small interval, which allows pruning of zero portions in the integral by examining the distance between sample points and $x_{o}$.

For single scattering, precomputing $\overrightarrow{S_{j}}\left(x_{o}\right)$ defined by Equation 15 requires integration along the negative normal direction of vertex $x_{o}$. We use 32 deterministic samples with importance sampling distribution function $\gamma(s)=e^{-s}$ along the path. The integration upper limit is the distance along the negative normal direction to the back side. Visibility is included by testing whether or not the incoming ray is occluded before entering the object. The precomputation is accelerated by applying the same pruning method in multiple scattering to polynomial basis $B_{j}^{E}$.

\subsection{Rendering}

As the user modifies the value of analytic BSSRDF parameters during an editing session, we first compute the polynomial coefficients $c_{j}^{R}, c_{j}^{E}$ using a least-square solver, and update the outgoing radiance at each vertex by multiplying the coefficients with the precomputed transport functions $T_{j}, \overrightarrow{S_{j}}$, as in Equations 9 and 14. Since human perceives glossy surfaces more translucent than surfaces without glossy, for increased realism, we add a specular component to the final rendering.

The system is further accelerated by exploiting GPU capabilities. After precomputation, we package all the vertex attributes, including positions and normals, as well as transport functions $T_{j}, \overrightarrow{S_{j}}$ into a $2 \mathrm{D}$ texture. During editing, we utilize the OpenGL render-to-vertex-array technique (including OpenGL extensions: vertex buffer object, pixel buffer ob- 
ject and framebuffer object ) and perform rendering in three steps. First, we compute polynomial basis coefficients $c_{j}^{R}, c_{j}^{E}$ in CPU and bind the coefficients to the shader program as uniform variables. Next, utilizing the uniform variables and the 2D texture, we render a rectangle to the frame buffer object, in which each pixel maintains the outgoing radiance of one vertex on the object. Finally, we copy the color result from frame buffer object to vertex arrays through pixel buffer object, and render the object using vertex buffer object.

\section{Comparison and Results}

We now present comparison tests of our method and example editing sessions in our system. The results are produced on a consumer-level PC with Intel Core2Duo 1.86G Hz processor, 2G RAM and a Nvidia GeForce 7800 256M graphics card. The rendering framerates, memory consumption and precomputation time for the scenes are reported in Table 2.

\subsection{Comparison}

In this section, we evaluate the approximation quality of our polynomial basis function and the resulting radiance computation in the context of alternative choices of basis and the analytic BSSRDF model proposed by Jensen et al. [JMLH01]. We will show that our approximation using piecewise linear basis with a small number of coefficients achieves satisfactory results.

In Figure 1, we compare approximations of $R_{d}$ using different basis functions, including Haar wavelet (used in [NRH03]), Daubechies wavelet (used in [BAOR06]), and our proposed piecewise linear and quadratic bases. Due to our non-uniform partitioning of intervals, our piecewise linear basis is able to approximate the strongly attenuated function $R_{d}$ using only 32 coefficients (16 intervals) with similar error as that of the full Daubechies 4 wavelet basis using 128 coefficients.

In Figure 2, we further reveal the approximation error of the piecewise polynomial basis to both $R_{d}$ and $E$ under different scattering and absorption coefficients $\left(\sigma_{s}\right.$ and $\sigma_{a}$ ). Note that, for larger $\sigma_{s}$ values, a greater number of polynomial coefficients (i.e., more intervals) is necessary to achieve an accurate approximation (since $R_{d}$ and $E$ attenuate more quickly). Nonetheless, the approximation errors decay rapidly with the growth of the number of coefficients. In particular, piecewise linear and quadratic basis result in extremely small error $(<0.005)$ when the number of coefficients exceeds 15 .

Finally, we compare in Figure 3 and 4 the outgoing radiance due to multiple scattering and single scattering approximated using our method to that computed by the analytic BSSRDF model, referred to as the ground truth. Observe that piecewise linear basis with 32 coefficients for multiple scattering and 16 coefficients for single scattering result in almost identical images with the ground truth. A smaller number of coefficients (e.g., 8 for multiple scattering, 6 for single scattering), however, may yield noticeable difference (e.g., the bottom of the bunny model and the face of the Caesar model), since the fewer intervals used in the polynomial basis approximation are incapable of accurately capturing the exponentially attenuated functions.

\subsection{Results}

Here we show examples of editing sessions using our system, where the user dynamically changes analytic BSSRDF parameters including the scattering coefficient (Figure 5), the absorption coefficient (Figure 6) and the phase function (Figure 7). Based on our observation in the comparison tests, piecewise linear basis with 32 coefficients for multiple scattering and 16 coefficients for single scattering are used in these examples (more coefficients or piecewise quadratic bases can be chosen in our system upon user requests).

Observe that the adjustment of analytic BSSRDF parameters results in change in the amount of translucency (Figures 5 and 6) as well as in backward or forward scattering effects (Figure 7). The ability to adjust these parameters dynamically with real-time rendering (see performance in Table 2) offers the designer timely feedback crucial for interactive applications, a convenience that has not been achieved previously.

\section{Conclusion and Future work}

In this paper, we proposed a method for real-time rendering of homogenous translucent material using Jensen's analytic BSSRDF model under fixed environment lighting and dynamically changing material parameters. Our system considers both multiple and single scattering components in the analytic model, allowing the user to change the scattering and absorption coefficients as well as the phase function interactively. Following the PRT rationale, we approximate the multiple scattering diffuse reflectance function and single scattering exponential attenuation function using nonuniform piecewise polynomial basis, which we have demonstrated to be better adapted to strongly attenuating functions than previously used bases.

While our method is currently restricted to Jensen's analytic model, we next plan to allow freely editing the $R_{d}$ and $E$ curves, and extend current method onto multi-layered and heterogenous materials. In addition, we will investigate ways to include dynamic lighting in our system. Last but not least, as the non-uniform piecewise polynomial basis excels in approximating fast-decaying functions, we are also interested in exploring applications in other areas of computer graphics where such approximation is useful.

Acknowledgements: We would like to thank the anonymous reviewers for their valuable comments. We would also like to thank Jiaping Wang for useful discussion. This work was partly supported by the National Basic Research Program of China (Project No. 2006CB303106), the National 


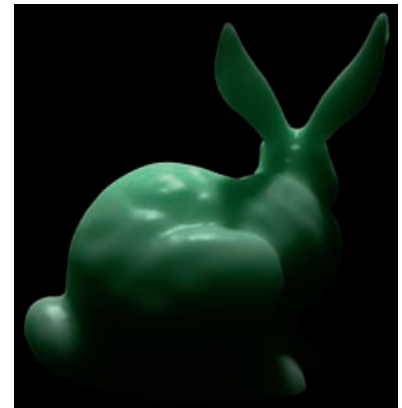

(a)

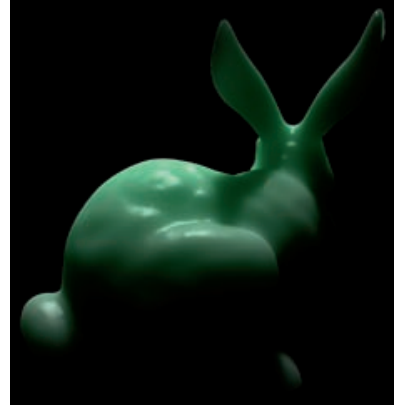

(b)

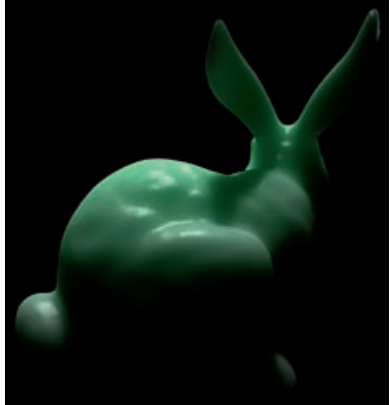

(c)

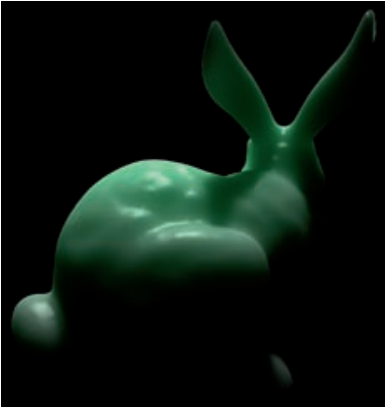

(d)

Figure 3: Comparison of subsurface scattering with only multiple scattering component: approximation by piecewise linear basis with 8 (a), 16 (b) and 32 (c) coefficients, and the ground truth image (d). Parameters $\sigma_{s}=[1.8,1.8,1.8]$ and $\sigma_{a}=$ $[0.03,0.004,0.03]$ are used.

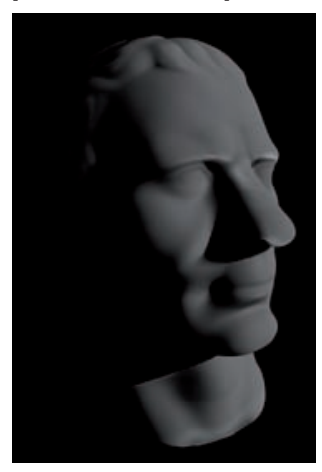

(a)

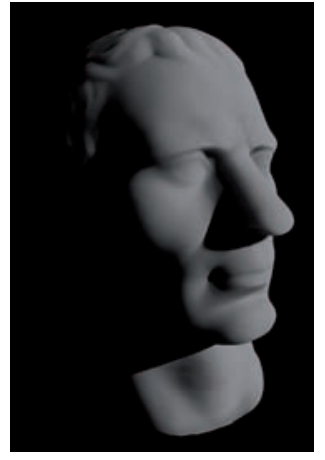

(b)

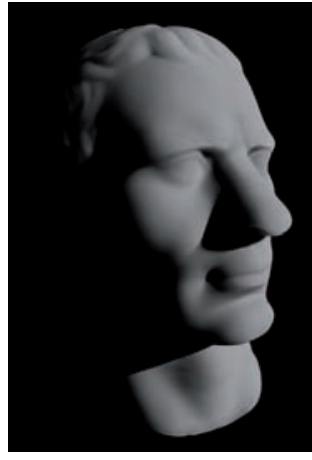

(c)

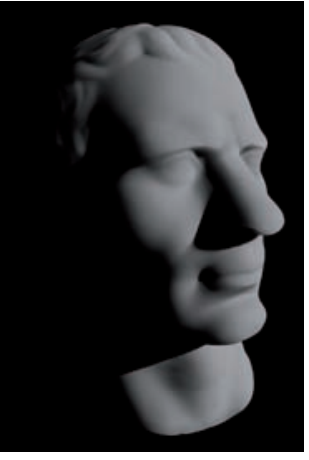

(d)

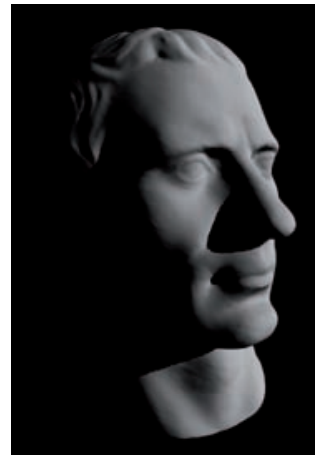

(e)

Figure 4: Comparison of subsurface scattering with only single scattering component: approximation by piecewise linear basis with $6(a), 8(b)$ and $16(c)$ coefficients, and the ground truth image (d). Parameters $\sigma_{s}=[1.0,1.0,1.0], \sigma_{a}=[0.01,0.01,0.01]$, and $g=0$ are used. A BRDF rendering is shown in (e) for reference.

High Technology Research and Development Program of China (Project Number 2006AA01Z304) and the Basic Research Foundation of Tsinghua National Laboratory for Information Science and Technology.

\section{References}

[BAOR06] BEN-ARTZI A., OVERBECK R., RAMAMOORTHI R.: Real-time brdf editing in complex lighting. ACM Trans. Graph. 25, 3 (2006), 945-954.

[DEL*99] Dorsey J., Edelman A., Legakis J., Jensen H. W., Pedersen H. K.: Modeling and rendering of weathered stone. In Proc. SIGGRAPH 1999 (1999), pp. 225-234.

[HK93] Hanrahan P., Krueger W.: Reflection from layered surfaces due to subsurface scattering. In Proc. SIGGRAPH 1993 (1993), pp. 165-174.

[HV04] HAO X., VARSHNEY A.: Real-time rendering of translucent meshes. In ACM Transactions on Graphics, vol. 23(2). ACM press, 2004, pp. 120-142.

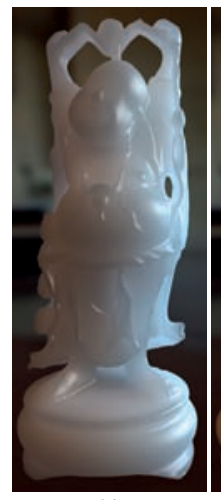

(a)

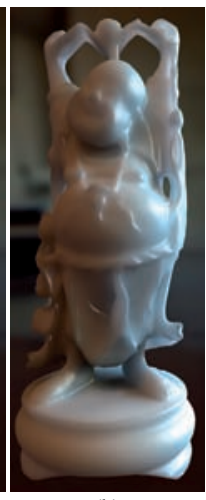

(b)

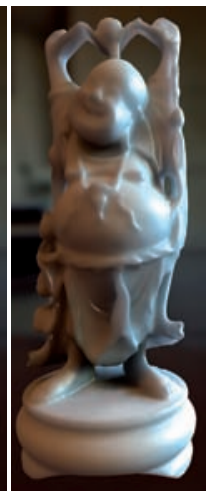

(c)
Figure 5: Editing the buddha model by increasing the scattering coefficient: $(a): \sigma_{s}=0.3, \sigma_{a}=0.01,(b): \sigma_{s}=$ 1.5, $\sigma_{a}=0.01,(c): \sigma_{s}=4.0, \sigma_{a}=0.01$.

[JB02] Jensen H. W., Buhler J.: A rapid hierarchical rendering technique for translucent materials. $A C M$ 


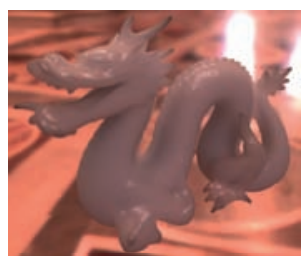

(a)

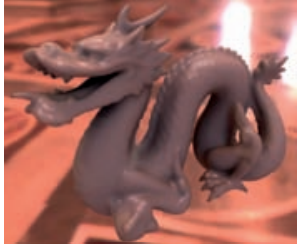

(b)
Figure 6: Editing the dragon model by increasing the absorption coefficient: $(a): \sigma_{s}=1.04, \sigma_{a}=0.002$, (b): $\sigma_{s}=$ $1.04, \sigma_{a}=0.40$.

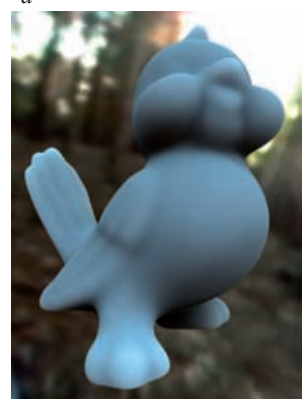

(a)

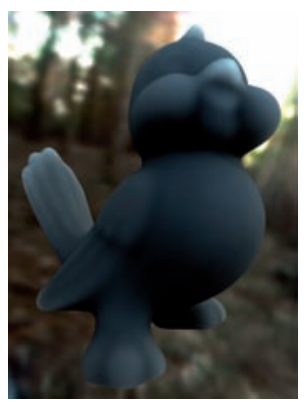

(b)
Figure 7: Editing the tweety model using the Eddington phase function with $g=-0.25$ (primary backward scattering) (a) and $g=0.25$ (primary forward scattering) (b).

\begin{tabular}{lcccc}
\hline Scene & Bunny & Buddha & Dragon & Tweety \\
\hline No. of Verts & $120 \mathrm{k}$ & $80 \mathrm{k}$ & $60 \mathrm{k}$ & $30 \mathrm{k}$ \\
MS Sample Pt. & $300 \mathrm{k}$ & $200 \mathrm{k}$ & $200 \mathrm{k}$ & $80 \mathrm{k}$ \\
MS P.T. & $118 \mathrm{~min}$ & $50 \mathrm{~min}$ & $35 \mathrm{~min}$ & $8 \mathrm{~min}$ \\
MS Storage & $23 \mathrm{M}$ & $15 \mathrm{M}$ & $12 \mathrm{M}$ & $6 \mathrm{M}$ \\
SS P.T. & $120 \mathrm{~min}$ & $84 \mathrm{~min}$ & $60 \mathrm{~min}$ & $32 \mathrm{~min}$ \\
SS Storage & $45 \mathrm{M}$ & $30 \mathrm{M}$ & $23 \mathrm{M}$ & $11 \mathrm{M}$ \\
MS FPS & 38 & 50 & 88 & 190 \\
Overall FPS & 16 & 26 & 37 & 70 \\
\hline
\end{tabular}

Table 2: Performance of results. Each column lists: the number of vertices, the number of sample points used for multiple scattering, precomputation time and storage for multiple and single scattering, framerates of only multiple scattering effects, framerates of both single and multiple scattering. For all these scenes, piecewise linear basis with 32 and 16 coefficients are used respectively for multiple scattering and single scattering.

Transactions on Graphics (Proceedings of SIGGRAPH 2002 Annual Conference) 21, 3 (2002), 576-581.

[JC98] Jensen H. W., Christensen P.: Efficient simulation of light transport in scenes with participating media using photon maps. In ACM SIGGRAPH (1998), pp. 311320.

[JMLH01] Jensen H. W., Marschner S. R., Levoy M., HANRAHAN P.: A practical model for subsurface light transport. In Proceedings of SIGGRAPH 2001 (August 2001), pp. 511-518.
[LGB*03] Lensch H. P. A., Goesele M., Bekaert P., Magnor J. K. M. A., Lang J., Seidel H.-P.: Interactive rendering of translucent objects. In Computer Graphics Forum, Duke D., Scopigno R., (Eds.), vol. 22(2). Blackwell Publishing Inc, Oxford, UK and Boston, USA, June 2003, pp. 195-205.

[LPT05] Li H., Pellacini F., Torrance K. E.: A hybrid monte carlo method for accurate and efficient subsurface scattering. In Rendering Techniques (June 2005), pp. 283-290.

[LRR05] LAWRENCE J., RUSINKIEWICZ S., RAMAMOORTHI R.: Adaptive numerical cumulative distribution functions for efficient importance sampling. In Eurographics Symposium on Rendering (June 2005).

[MKB*03] Mertens T., Kautz J., Bekaert P., SeiDEL H.-P., REETH F. V.: Interactive rendering of translucent deformable objects. In Proceedings of the 14th Eurographics workshop on Rendering (Leuven, Belgium, 2003), Eurographics Association, pp. 130-140.

[NRH*77] Nicodemus F. E., Richmond J. C., HsiA J. J., Ginsberg I. W., Limperis T.: Geometrical Considerations and Nomenclature for Reflectance. National Bureau of Standards (US), 1977.

[NRH03] NG R., RAmamoorthi R., HanRAhan P.: All-frequency shadows using non-linear wavelet lighting approximation. ACM Trans. Graph. 22, 3 (2003), 376381.

[PH00] Pharr M., Hanrahan P. M.: Monte Carlo evaluation of non-linear scattering equations for subsurface reflection. In Proc. SIGGRAPH 2000 (2000), pp. 275-286.

[SHHS03] Sloan P.-P., Hall J., Hart J., SNyder J.: Clustered principal components for precomputed radiance transfer. ACM Trans. Graph. 22, 3 (2003), 382-391.

[SKS02] Sloan P.-P., Kautz J., Snyder J.: Precomputed radiance transfer for real-time rendering in dynamic, low-frequency lighting environments. In Proceedings of Siggraph 2002 (2002), ACM Press, pp. 527-536.

[SLS05] SloAN P.-P., LunA B., SNyder J.: Local, deformable precomputed radiance transfer. ACM Trans. Graph. 24, 3 (2005), 1216-1224.

[Sta95] Stam J.: Multiple scattering as a diffusion process. In Eurographics Rendering Workshop (June 1995), Eurographics, pp. 41-50.

[TS06] Tsai Y.-T., SHIH Z.-C.: All-frequency precomputed radiance transfer using spherical radial basis functions and clustered tensor approximation. In Proceedings of SIGGRAPH 2006 (2006).

[WTL05] WANG R., TRAN J., LuebKe D.: Allfrequency interactive relighting of translucent objects with single and multiple scattering. ACM Transactions on Graphics 24, 3 (July 2005), 1202-1207. 